\title{
An endoscopic multi-exposure laser speckle contrast analysis system for blood flow and microcirculation measurements
}

\author{
M. Tziraki, L. Song ${ }^{*}$, D. S. Elson \\ The Hamlyn Centre for Robotic Surgery, Department of Surgery and Cancer, Imperial College \\ London, UK. \\ * Current address: College of Electronic Information and Optical Engineering, Nankai University, \\ China.
}

\begin{abstract}
We present a dual wavelength endoscope which uses Endoscopic Laser Speckle Contrast Analysis (ELASCA) with the aim to image tissue blood flow and perfusion during surgical procedures. In this study we measure speckle decorelation times, which are associated with flow, by imaging speckle patterns at a wide range of detector exposure times. In order to understand the effects of image collection efficiency and sample scattering properties, control experiments with different optical systems were performed by imaging of tissue mimicking phantoms and inferring their flow parameters.
\end{abstract}

Keywords: Speckle contrast, blood flow, endoscopy.

\section{INTRODUCTION}

Imaging tissue microcirculation and blood perfusion indices can aid diagnosis at a number of conditions such as stroke, ischemia, cancer, and cardiovascular abnormalities and can also help assessments of organ viability during surgical transplant procedures. A relatively simple optical imaging technique is Laser Speckle Contrast Analysis (LASCA), which was introduced by Fetcher and Briers [1] in 1981 to image blood flow. The technique has been used in the past decade, at preclinical studies of blood flow imaging in the brain and the retina; it is also used to image skin perfusion. Using laser illumination on the tissue, the movements of the light-scattering blood cells cause a fluctuating granular pattern called speckle in the image. Applying statistical analysis, the contrast values of the speckles are calculated and a speckle contrast image is produced. The blood flow distribution is visualized in a qualitative way as changes of the speckle contrast, the lower the contrast the higher the flow. There is an inversely proportional relationship between speckle contrast, and speckle decorrelation times with flow velocity. Parthasarathy et al. [2] proposed the idea of using Multiple Exposure durations to obtain Speckle contrast Imaging (MESI) to measure flow velocities in microfluidic phantoms. Quantitative speckle contrast imaging is increasing being investigated, since then, mainly in brain blood flow monitoring using microscopic imaging set ups commonly implemented with objective lenses.

To increase the application areas of LASCA, an endoscopic set up was implemented by Song et al. [3], in order to image the vasculature of internal organs such as colon wall and oesophagus in standard endoscopic clinical procedures and to assess the viability and health of organs during transplant surgery. ELASCA could be used as a qualitative tool to indicate flow maps. In this study we examine the quantitative aspects of this instrument. To address this issue we apply the principles of MESI to image flow in phantoms with non-scattering and scattering medium. We compare the results with ones from a microscope set up.

\section{METHODOLOGY}

The theory behind the multiple exposure model has its roots in Fetcher and Briers [1] and Bandyopadhyay et al. [4] and is an extension of mapping the speckle contrast over a range of camera exposure times to achieve a more quantitative set of flow merits accounting for the effect of static scatters. In the MESI model the speckle contrast (K) is related to the camera exposure duration $(\mathrm{T})$ and speckle correlation time $\left(\tau_{\mathrm{c}}\right)$ as follows: 


$$
K\left(T, \tau_{c}\right)=\left\{\beta \rho^{2} \frac{e^{-2 x}-1+2 x}{2 x^{2}}+4 \beta \rho(1-\rho) \frac{e^{-x}-1+x}{x^{2}}+v_{n e}+v_{n o i s e}\right\}^{1 / 2}
$$

Where $\mathrm{x}=\mathrm{T} / \tau_{\mathrm{c}} \beta$ is a normalization factor to account for speckle spatial sampling, $\rho$ is the fraction of light which is dynamically scattered, $\rho=\mathrm{I}_{\mathrm{f}} /\left(\mathrm{I}_{\mathrm{f}}+\mathrm{I}_{\mathrm{s}}\right)$, f indicates dynamic scatterers and s static scatterers, $v_{\text {ne }}$ is a term associated with non ergodic light and $v_{\text {noise }}$ is noise from the detector. The speed $\mathrm{v}$ of the scattering particles is inversely proportional to $\tau_{\text {c. }}$ Our endoscopic instrument is designed to image the organ, blood flow and perfusion and potentially to extract information on oxygenation and metabolism; therefore a spectroscopic modality was added by employing lasers at two illumination wavelengths.

The probe of the dual illumination laser speckle contrast endoscopic imaging system (Fig.1) includes two polarisation maintaining fibres (PM fibre, Thorlabs ) that relay the light from two lasers diodes (ML101J27 660nm and DL8142 830 $\mathrm{nm}$ Thorlabs) onto the tissue. A plano-convex lens ( $\mathrm{f}=6 \mathrm{~mm}, \mathrm{D}=3 \mathrm{~mm}$, Edmund Scientific) was placed between a polariser and the near end of a leached fibre image guide (LFIG). The probe diameter is $7.7 \mathrm{~mm}$ and its length is $2 \mathrm{~m}$. The image transmitted through the (LFIG) was imaged onto a cooled 12 bit CCD at a chip size $8.9 \times 6.7 \mathrm{~mm}^{2}$, after magnification of $\mathrm{x} 7$ with a microscope objective (10 x, Olympus) and an achromatic lens ( $\mathrm{f}=100 \mathrm{~mm}$, Thorlabs). A neutral density (ND) filter was placed at the detection path, when needed to control the intensity and keep the equal detection intensity requirement. The two lasers were switched on and off alternately and were synchronised with the camera's acquisition via a data acquisition (DAQ) card (National Instruments) and controlled by a LabVIEW program. Images were slightly defocused from the honeycomb pattern of the LFIG and were acquired at both wavelengths up to the speed of 36 fps.

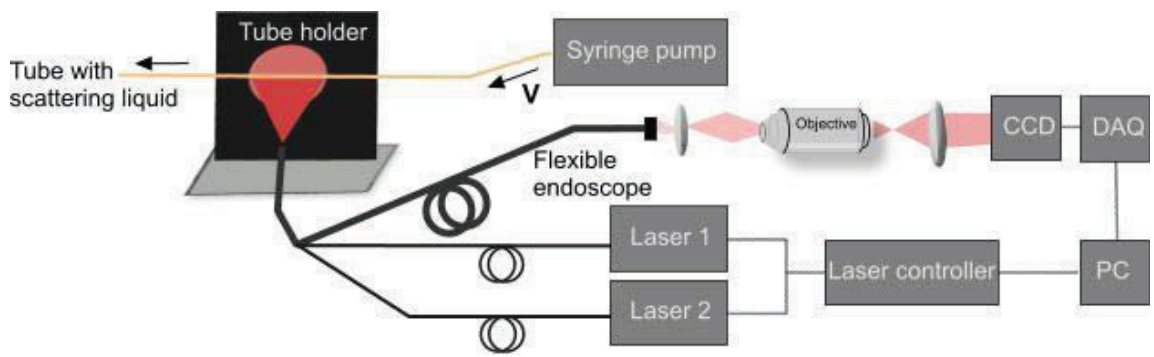

Figure 1. The experimental set-up, employing a flexible fibre image guide to image flow speed of a scattering liquid in a tube.

The experiment presented here consists of three parts: the first part was performed using an endoscopic probe to image pure flows of liquid in a tube in order to measure the speckle decorrelation time, the second part aimed to measure the decorrelation times when multiple scattering was present at the object plane. In the third part, the tube was imaged using an $10 \mathrm{x}$ objective, in free space, instead of using the fibre image guide probe. This was done to try to understand, the effect of the fibre image guide and to compare the results with the literature [2]. Experimental data was recorded using a phantom consisting of a $1 \mathrm{~mm}$ hollow diameter silicon tube above a black non-scattering substrate. The tube was connected to a mechanical syringe pump (World Precision Instruments, Saratosa, FL, USA) and a suspension of $1 \mu \mathrm{m}$ polystyrene microspheres in water with $\mu_{\mathrm{s}}{ }^{\prime}=3 \mathrm{~mm}^{-1}$ was pumped into it, at controlled flow speeds. Data was recorded at ten exposure durations with span ranging from $0.1 \mathrm{msec}$ to $100 \mathrm{msec}$ and five flow velocities ranging from $1 \mathrm{~mm} / \mathrm{sec}$ to $5.25 \mathrm{~mm} / \mathrm{sec}$. For each flow velocity, the camera exposure duration was varied and 60 images of the tube were recorded for each exposure time. To examine the effects of multiple dynamic scattering, the experiment was repeated using a 1.5 $\mathrm{mm}$ thickness, silicone based scattering substrate, where scattering was induced by adding $1 \mu \mathrm{m} \mathrm{SiO}_{2}$ microspheres giving $\mu_{\mathrm{s}}{ }^{\prime}=1 \mathrm{~mm}^{-1}$. For each exposure time, a constant intensity was maintained using ND filters at the detection path; this was a requirement to ensure contrast values were less affected by the intensity variations of the speckle pattern.

\section{RESULTS}

The speckle contrast map of each recorded image was calculated and presented at Fig. (2). A region of interest (ROI), which covered the flow area, was selected and the mean contrast value within this ROI was calculated for the duration of each exposure time. 


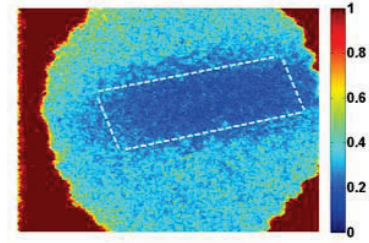

$0.5 \mathrm{msec}$

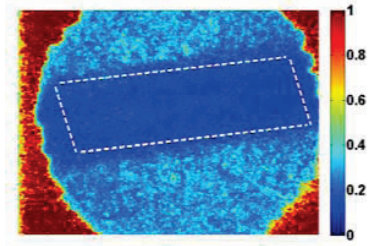

$5 \mathrm{msec}$

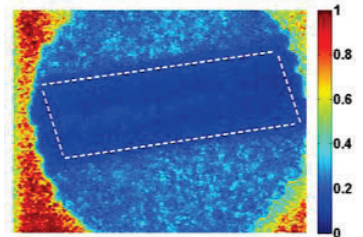

$20 \mathrm{msec}$

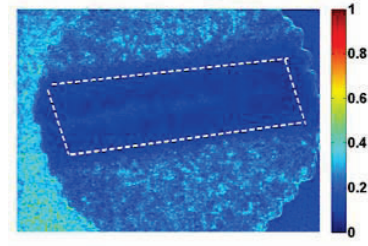

$70 \mathrm{msec}$

Figure 2: Speckle contrast map images of the silicone tube with solution flowing at $2 \mathrm{~mm} / \mathrm{sec}$ for various camera exposure durations. The imaging area at the object plane had a diameter of $2.5 \mathrm{~mm}$. The ROI where the average contrast is calculated is indicated by the rectangular in each image.

The distribution of the speckle contrast over the exposure duration was plotted at flow velocities of $3.18 \mathrm{~mm} / \mathrm{sec}$ and $5.25 \mathrm{~mm} / \mathrm{sec}$. For clarity only three from the five recorded velocities distributions are presented.

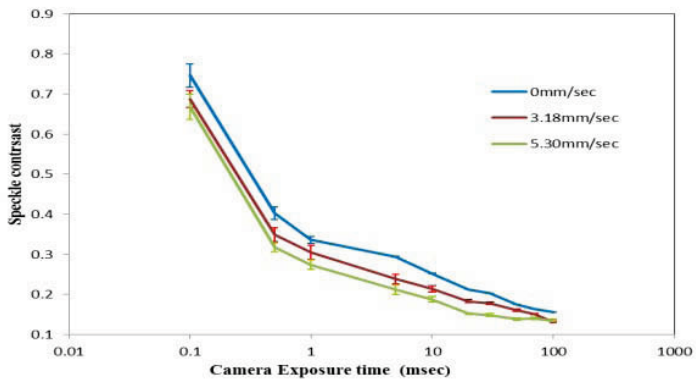

(a)

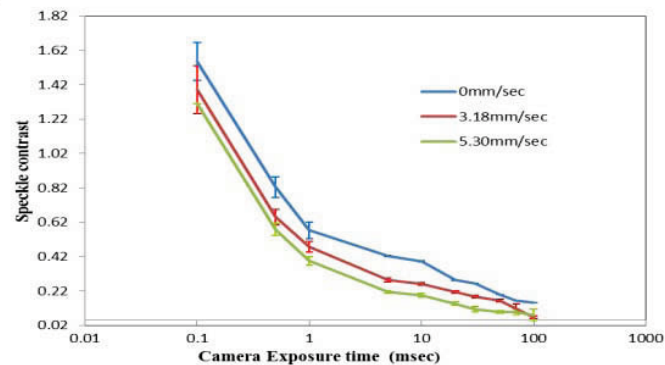

(b)

Figure 3. The variation of speckle contrast with exposure duration for various flow speeds recorded at (a) $660 \mathrm{~nm}$ and (b) $830 \mathrm{~nm}$ with the endoscope. The error bars represent the standard deviation of measurement within the selected ROI.

As is shown in Fig (3) there are some differences in the speckle contrast values between red and infrared illumination. The higher contrast values in the IR region could be an effect of the deeper penetration. Moreover there is a high deviation at $100 \mu \mathrm{sec}$ exposure duration from the expected value which is less than 1 . At this small exposure duration it was not possible to maintain the same mean intensity as for the other measurements and the resulting data is unexpectedly high and could be influenced by noise. The error bars of the distribution at low exposure times are high indicating the measurement to be inaccurate at $100 \mu \mathrm{sec}$ exposure duration where the recorded intensity was very low, which was also observed by Song et al [5]. Over all the distribution between the two wavelengths are similar.

The data from each curve were fitted to equation (1) and the $\tau_{\mathrm{c}}$ along with the other parameters were calculated from the best fit. The results for the distribution at $3.18 \mathrm{~mm} / \mathrm{sec}$ flow speed are presented in Fig (4), in the presence and absence of a scattering substrate. With the scattering substrate the distribution is elevated as the photons experience multiple stationary scattering events resulting in high speckle contrast values. By fitting the data the $\tau_{\mathrm{c}}$ could be found as well as the flow average speed which is inversely proportional to $\tau_{\mathrm{c}}$. The calculated velocities were different from the velocities, according to the syringe pump, by 22-35\%. Possible reasons from this are:(a) the defocusing effect, compared to imaging with an objective, (b) the silicone tube walls of thickness of $300 \mu \mathrm{m}$, which could introduce distortions in the speckle pattern, (c) the effect of the low light intensity for the low exposure durations. Here the original data is presented without any corrections for low intensity and its influence on contrast, proposed by Song et al [5].

The speckle contrast map of the same tube recorded with the objective lens instead of the endoscopic probe is shown in Fig (5a). The red area at the bottom of the image is the non-scattering substrate. The tube walls are clearly visible and are yellow-green colour and they are $300 \mu \mathrm{m}$ wide, (b) is the speckle contrast variation with exposure times. Equation (1) was fitted to the distributions of Figure $(5 \mathrm{~b})$ to allow the calculation of $\tau_{\mathrm{c}}$, and the flow velocities. 


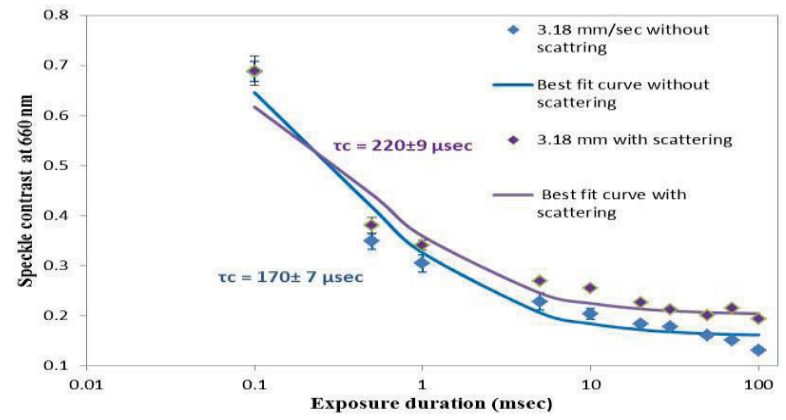

Figure 4: The fitting of Equation (1) to the recorded fibre image guide ELASCA data for a flow speed of $3.18 \mathrm{~mm} / \mathrm{sec}$ at $660 \mathrm{~nm}$. The error bars represent the standard deviation of measurement within the selected ROI

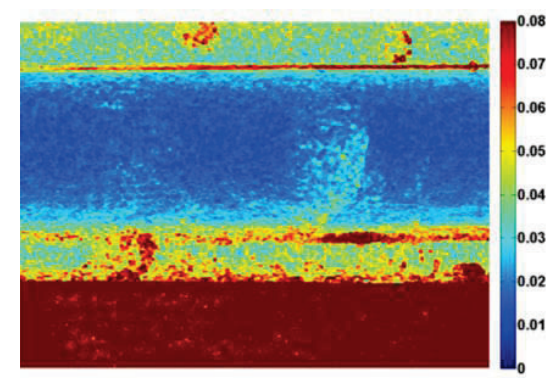

(a)

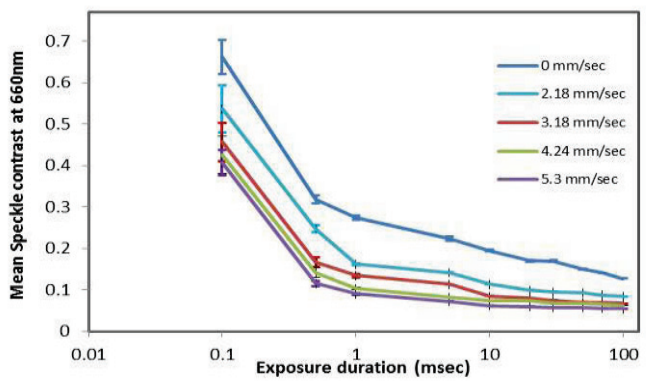

(b)

Figure 5. (a) A single image of the speckle contrast map of the tube recorded with the $x 10$ objective for liquid flow speed of $2.18 \mathrm{~mm} / \mathrm{sec}$ and exposure duration of $20 \mathrm{msec}$. (b) Speckle contrast variation with exposure time using the objective. . The imaging area at the object plane had dimensions of $2 \times 2.5 \mathrm{~mm}^{2}$. The error bars represent the standard deviation of measurement within the ROI where the speckle contrast was calculated.

\section{CONCLUSIONS}

The ability to calculate flow velocities with MESI while imaging with a dual wavelength endoscopic probe was explored. The system was used to image an area where scattering fluid flowed through a $1 \mathrm{~mm}$ hollow diameter silicone tube. Flow speeds were calculated with an error varying from $22 \%$ for a non-scattering and $30 \%$ for a scattering background.

\section{ACKNOWLEDGMENTS}

M. Tziraki gratefully acknowledges funding from EU FP7 via IEF Marie Curie Fellowship scheme. Finding for this project was provided by ERC 242991.

\section{REFERENCES}

[1] A. F. Fetcher and J. D. Briers, "Flow visualization by means of single-exposure speckle photography," Opt. Commun. 37, 326-330 (1981).

[2] A. B. Parthasarathy, W. J. Tom, A. Gopal, X. Zhang, and A. K. Dunn, "Robust flow measurement with multiexposure speckle imaging," Opt. Express 16(3), 1975-1989 (2008).

[3] L. Song and D Elson "Endoscopic laser speckle contrast imaging system using a fibre image guide" Proc. of SPIE Vol.7907 79070F-10F-1 (2011).

[4] R. Bandyopadhyay, A. S. Gittings, S. S. Suh, P. K. Dixon, and D. J. Durian, "Speckle-visibility spectroscopy: A tool to study time-varying dynamics," Rev. Sci. Instrum. 76, 093110 (2005).

[5]L Song D S Elson 'Effect of signal intensity and camera quantization on laser speckle contrast analysis'”Biom. Opt.Exp 4 (1)89-104 (2013). 